\title{
Gently handled foals generalize responses to humans
}

Anita Schmidek - Bruno Nogueira de Oliveira -

Pedro Trindade - Mateus José Rodrigues Paranhos da Costa

A Schmidek (Corresponding author)

Polo Regional de Desenvolvimento Tecnológico dos Agronegócios da Alta Mogiana, Colina, SP, Brasil, Brazil. email: anita@apta.sp.gov.br
BN Oliveira - P Trindade - MJR Paranhos da Costa

Grupo de Estudos e Pesquisas em Etologia e Ecologia Animal (ETCO), Faculdade de Ciências Agrárias e Veterinárias da Unesp, Campus de Jaboticabal, SP, Brazil.

Received: June 27, 2017 • Revised: September 22, 2017 • Accepted: September 22, 2017

\begin{abstract}
Equines perform tasks along humans, and there are evidences and controversies that they are able to discriminate between familiar and unfamiliar humans. This study assessed whether foals can discriminate between humans in terms of familiarity and human experience in equine handling. Daily, in the first two weeks of life, 30 foals went through a short section of gentle handling. After about four months, a human forced approach test was carried out by 4 evaluators varying in the familiarity aspect and experience with equine handling. Data was submitted to the McNemar test $(\mathrm{P}<0.05)$. In $66.7 \%$ of the assessments, the foals accepted the human approach, and among the positive cases, $60.0 \%$ were characterized by seeking contact with the evaluators. Among the foals that accepted the human approach, 97.5\% also accepted tactile stimulation. No differences were found regarding the familiarity of the evaluators $(\mathrm{P}>0.05)$ or their experience in equine handling $(\mathrm{P}>0.05)$. The individual variation was evident, which indicates that we must work with personalized training techniques, in which the behavior of each individual is the main factor to be considered. Our results show that the adoption of good handling practices with foals favored their relationship with humans, and it is possible to assume that such practices can improve animal welfare, as well as the safety of the humans that will have contact with them in the future.
\end{abstract}

Keywords: animal welfare, equines, training

\section{Introduction}

Horses perform tasks that depend on a harmonious human-horse relationship as for equestrian sports, farm work, transportation and others. Most of the equine population of United States (Kilby 2007) and Brazil (Lima et al 2006) are in farm work, sports and leisure, as it happens in Europe where urban use of horses increased during the last decade with over 15,000 equestrian facilities running in 2009 (Liljenstolpe
2009). This indicates a growing intensification of humanhorse interactions.

Creating social bonds is an important aspect in equine behavior. It can be developed as intra-species interactions, and also among different species, such as humans and horses (Goodwin 1999). Therefore, comprehension of equine behavior is important to establish positive interactions between horses and humans. Creating these social bonds interferes directly in the way horses react to our presence, which involves mutual influences between horses' temper and the sum of experiences of both - horses and humans (Hausberger et al 2008). Some studies using different species show the importance of previous experiences in defining reaction of animals toward humans, ex. cattle (Lewis and Hurnik 1998), ovines and swines (Oliveira 2003; Oliveira et al 2015) and horses (Søndergaard and Halekoh 2003). However, it is not clear yet which factors may interfere in the duration of such effects in animals (Waiblinger et al 2006).

Evidences suggest that animals of various species including horses, are capable of discriminating humans (Tanida and Koba 2001; Waiblinger et al 2006; Stone 2010). However, it is controversial if animals may respond the same way to familiar or unfamiliar humans in different behavioral tests (Tanida et al 1995; Hemsworth et al 1994; Simpson 2002; Henry et al 2005). Besides, the influence of factors such as the experience of the evaluator with equine handling and human body posture during behavioral assessments still raises questions (McKinley and Sambrook 2000; Chamove et al 2002; Seaman et al 2002). The present study aims to assess the capability of foals in discriminating between familiar and unfamiliar humans and between humans with or without handling experience.

\section{Materials and Methods}

The study was conducted at Polo Regional de Desenvolvimento Tecnológico dos Agronegócios da Alta Mogiana, in Colina City, São Paulo State, Brazil. Thirty foals 
(12 males and 18 females, average age of 2.5 $\pm 1,5$ months-old) being 26 horses (Brazilian showjumping $n=23$ and Postier Breton $\mathrm{n}=3$ ) and 4 Brazilian Donkey where behaviorally assessed. All foals were nursing and kept on pasture with their mothers.

Daily, for the first two weeks, each foal and mare were sequentially conducted to a 64 sq. meter pen in order to have the navel treated. No other animals were in the pen. During this handling, mares were fed commercial feed $(1 \mathrm{~kg})$.

Handling was performed by the same person, identified as evaluator 1 (E1). Handling procedures were standardized as follows: E1 approach was always by the front of foal, keeping a $45^{\circ}$ angle to the longitudinal axis of the animal's body, slowly and without any abrupt movements, with shrunken shoulders, relaxed posture, looking straight to the foal but without starring in the eyes. E1 vocalized in low tone without characterizing a command. When near the foal, the evaluator touched the animal's shoulder with the back of the hand and then positioned next to the animal. Then the animal was gently restrained by the evaluator, who placed one arm around the foal's chest and the other arm over the hock, hugging it. Tactile stimulation was performeed as the evaluator gently patted the hand over the animal's body with gradual increase of intensity throughout the sections. The increase in the intensity of stimulation was carried out according to foal's reactions, preventing it to get tense or scared. Then the navel was treated using iodine solution. During this procedure, if the foal attempted to move, E1 tried to contain it, increasing the pressure of the arms and kept this procedure until the foal stopped this attempt. At this time, the arm pressure was released. Handling procedure lasted 10 minutes maximum per day and sections were never concluded if the foal was unquiet or attempting to run away. If necessary, a few minutes were spent until the foal has calmed down, before letting go.

The assessment of foals' behavior was based in the Forced Human Approach test (FHA test) proposed by Søndergaard and Halekoh (2003). The test consisted in the evaluator's attempt to approach each foal by four different human evaluators performing the test, where Evaluator 1 (E1 - familiar and with handling experience), Evaluators 2 and 4 (E2 and E4 - unfamiliar and without handling experience) and Evaluator 3 (E3 - unfamiliar with handling experience). In this test, each animal was assessed four times. The order in which the evaluators approached each foal was randomly defined with equal numbers of animals being evaluated in first, second, third and fourth place by each of the evaluators. The animals were allocated together in a pasture with 3 ha. All evaluators used the same approach technique as described above.

In order to assess the process of generalization to the stimuli (familiar or unfamiliar human and experienced or inexperienced), the foals received scores according to their reaction during the forced approach test, being assigned the score "A" to the animals that walked towards the human during the approach or that were standing still when the human approached, and the score "B" to the animals that did not allow the approach of the evaluator, fleeing and therefore, preventing the tactile stimulation. In addition, the foals that received the "A" score were assessed for their response to tactile stimulation, being classified as "positive" when they allowed tactile stimulation and "negative" when they did not allow (ex, animal walked while evaluator attempted to perform tactile stimulation was classified as "negative"). After the evaluations, a classification was established for the foals according to the consistency of their reactions to the approach of the four evaluators, defining the category "stable" for the foals that received the same scores by the four evaluators or "unstable" when they received at least one different score.

Data from the forced approach test ("A" or "B") and the acceptance of tactile stimulation ("positive" or "negative") were submitted to the McNemar test $(\mathrm{P}<0.05)$ to verify the existence of the association between the evaluators and the foals' response. All statistical analysis were performed with SAS $^{\circledR} 9.3$ statistical package (SAS Inst. Inc., Cary, NC).

\section{Results and Discussion}

In $66.7 \%$ of the forced approach tests, the foals accepted the human approach (score "A"), and in these cases, $60.0 \%$ had an active behavior to seek contact with the evaluators (see Figure 1). Among the foals that accepted the human approach, $97.5 \%$ also accepted tactile stimulation. No significant differences were found in the reactions of the animals during the forced approach test and acceptance of tactile stimulation between familiar and unfamiliar evaluators $(\mathrm{P}>0.05)$. Likewise, there was no significant difference between evaluators with and without equine handling experience $(\mathrm{P}>0.05)$. Regarding the behavioral stability towards the four evaluators, $46.7 \%$ of the foals presented "A" scores for all evaluators during the forced approach test and $20.0 \%$ of the foals presented a "B" score for the four evaluators. These results evidenced the efficiency of the previous handling sessions due to the high percentage of animals that allowed the approach and manipulation by the evaluators, although some of them were unknown to the foals and without prior experience in equine handling.

Our results show that foals generalize the response between familiar and unfamiliar humans, which agrees with several studies (Hausberger and Muller 2002, Henry et al 2005, Ligout et al 2008). In common, these studies had the use of forced approach tests in horses. Henry et al (2005) used positive tactile stimuli only directed at the dams, whereas in our study the familiar human performed tactile stimuli directed to the foals, stimuli that could be neutral, positive or negative (restraint with pressure) depending on the reaction of 
the foal. On the other hand, the study of Hausberg and Muller (2002) used adult animals in sports training and it was assumed that there were positive and negative stimuli.

Hemsworth et al (1994) presented similar results with piglets submitted to handling carried out by two handlers in a commercial industry. In this study, piglets from two groups which received negative stimuli or positive stimuli prior to the test - were unable to discriminate between humans in forced approach tests based on their previous handling experience. In another study conducted by the same group (Hemsworth et al 1996), the researchers found results on the generalization of cattle and swine stimuli, regarding regular or irregular exposure of humans or a new object. Thus, based on the results of these studies, it is possible to conclude that there is a generalization of animal stimuli in relation to humans, regardless the type of stimulus (positive or negative) received in daily handling for the animal species studied.

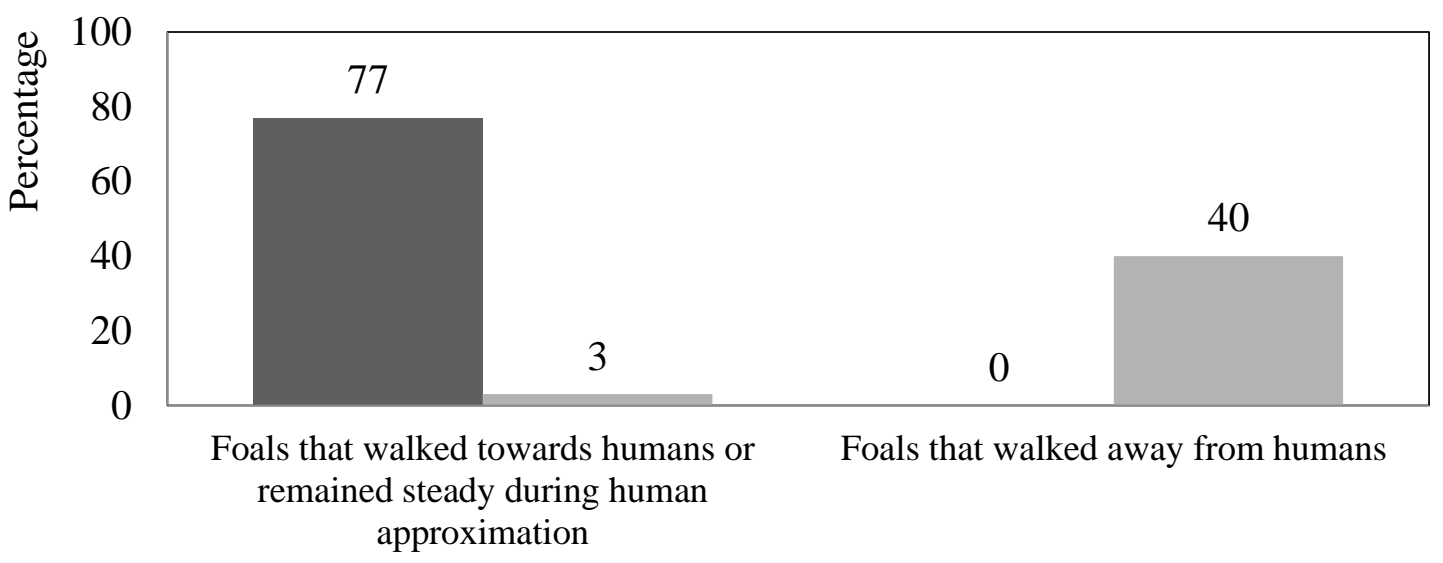

Foals that accepted tactile stimulation $\square$ Foals that did not accept tactile stimulation

Figure 1 Percentages of foals that accepted and did not accept tactile stimulation in any part of their bodies, within the group of foals that walked towards humans or remained steady during human approximation, and the group of foals that walked away from humans.

However, these findings cannot be generalized since there are studies with cattle (De Passillé et al 1996; Breuer et al 2003) that refute this assertion. The authors verified that animals that experienced negative stimuli differentiated the human responsible for this stimulus. We speculate that the results obtained in these studies may be due to the intensity and/ or duration of the negative stimuli, which may have contributed to the strong association between humans and negative stimulation, causing more significant changes in the animals' behavior. In fact, the study of De Passillé et al (1996) demonstrated behavioral changes influenced by the type and duration of the stimulus received by the animal. Based on their results, the authors concluded that after the animals experienced a period of negative stimuli, their behavior changed as the duration was influenced by the constancy of a new treatment, with positive stimuli. In this case, it is possible that a new association with other stimuli occurred without replacing the association of the initial stimuli, as described by Balkenius (2000). Depending on the duration and/ or intensity of a given stimulus, classical habituation or conditioning may occur. These are distinct processes that may be important when assessing animal reactions to humans.
Balkenius (2000) defines habituation as a learning process in which the animal learns to ignore a stimulus that does not represent anything important (neither positive nor negative). Classical conditioning is based on the pairing of two stimuli, being generally one neutral stimulus while the other represents something positive or negative to the animal (Mackintosh 1983). It seems reasonable that, when we consider neutral experiences and positive or negative experiences of low intensity which the predominant process is habituation, the generalization of animal responses to humans may occur more stable among animals.

Jago et al (1999) emphasize the importance of stimulus intensity as a modulating factor for animal behavior by showing that the food reward is a positive reinforcement of greater intensity for cattle when compared to the tactile stimulus. It was shown that the stimulus of greater intensity caused behavioral changes, whereas the weaker stimulus did not modify the reactions of animals to humans. The authors classified the tactile stimulus as a neutral stimulus and, therefore, the animals would undergo a process of habituation and not conditioning. On the other hand, Tanida et al (1995) described a differentiation of pig behavior between familiar and unfamiliar humans based only on tactile stimuli. It should 
be noticed that this study was developed with recently weaned piglets, therefore, with animals in a state of psychological stress. In addition, the familiar evaluator used a gentle daily handling (behavioral pre-test) characterized by the absence of restraint or forced approach to animals. We speculate, in this specific context, that tactile stimuli may have assumed a positive and more intense character. Therefore, classical conditioning was predominant, creating a strong association between the presence of the familiar human and positive or neutral sensations to the animals. In this perspective, in environments where the animal does not feel strongly threatened or rewarded, we assume that generalization occurs to stimuli received by the animals, in relation to humans. However, this may not be valid for situations in which negative or positive stimuli of greater intensity occur during the daily handling of the animals.

Standardization of the body posture and the approach method of the human during the behavioral test is another crucial factor to be considered in generalization studies. There are reports that an aggressive human posture and direct visual contact may provoke more intense fear reactions in horses (Miller 1995). However, these reports were not confirmed by the results of Seaman (2002), who found no relation between direct eye contact and horse behavior. We did not find studies that assessed the equine reaction to the forced approach of humans at different speeds and trajectories (straight or zigzag). In any case, as reported by Exadaktylos (2002), one should bear in mind that the forced approach to animals may be responsible for the cause of accidents among humans and horses. In view of the contradictions in the results of these studies, it is reasonable to assume that the standardization of the approach method between the different evaluators in the present study may explain the absence of significant differences in foal reactions to the forced approach of humans with and without experience on equine handling.

\section{Conclusions}

Individual variation in the responses to the tests was evident since some animals did not allow the approach and handling by the evaluators. This indicates the need to adopt personalized training techniques, in which the behavior of each individual is the main factor to be considered.

Our results show that the adoption of good handling practices in the initial phase of the foals' life favored the establishment of positive bonds with humans. In addition, the foals presented generalization to the stimuli received by humans, and it is possible to assume that such practices can improve the welfare of the animals, as well as the safety of humans who will have contact with them in the future, since there is less risk of aversive interactions between humans and equines.

\section{Acknowledgment}

We would like to thank Alcilany Nascimento Paiva, João Marcos Pereira Rios and Flávio Kikuichi (in memorian) for participating at the data collection. We also would like to thank the research support of Luiz Carlos Tomaz Ferreira.

\section{References}

Balkenius C (2000) Attention, habituation and conditioning: toward a computational model. Cognitive Science Quarterly 1:171-214.

Breuer K, Hemsworth PH, Coleman GJ (2003) The effect of positive or negative handling on the behavioural and physiological responses of nonlactating heifers. Applied Animal Behaviour Science 84:3-22.

Chamove AS, Crawley-Hartrick OJE, Stafford KJ (2002) Horse reactions to human attitudes and behaviour. Anthrozoos 15:323-331.

Exadaktylos A, Eggli S, Inden P, Zimmermann H (2002) Hoof kick injuries in unmounted equestrians. Improving accident analysis and prevention by introducing an accident and emergency based relational database. Emergency Medicine Journal 19: 573-575.

Goodwin D (1999) The importance of ethology in understanding the behaviour of the horse. Equine Veterinary Journal 28:15-19.

Hausberger M, Roche H, Henry S, Visseret EK (2008) A review of the human-horse relationship. Applied Animal Behaviour Science 109:1-24.

Hemsworth PH, Coleman PJ, Cox J, Barnett JL (1994) Stimulus generalization: the inability of pigs to discriminate between humans on the basis of their previous handling experience. Applied Animal Behaviour Science 40:129-142.

Hemsworth PH, Price EO, Borgwardt R (1996) Behavioural responses of domestic pigs and cattle to humans and novel stimuli. Applied Animal Behaviour Science 50:43-56.

Henry S, Hemery D, Richard MA, Hausberger M (2005) Humanmare relationships and behaviour of foals toward humans. Applied Animal Behaviour Science 93:341-362.

Jago JG, Krohn CC, Matthews LR (1999) The influence of feeding and handling on the development of the human-animal interactions in young cattle. Applied Animal Behaviour Science 62:137-151.

Kilby ER (2007). The demographics of the U.S. equine population. In: Salem DJ, Rowan AN (Eds.) The state of the animals Humane Society Press, Washington, DC, pp. 175-205.

Liljenstolpe C (2009) Horses in Europe. Uppsala: Swedish University of Agricultural Sciences.

Lewis Nj, Hurnik JF (1998) The effect of some common management practices on the ease of handling of dairy cows. Applied Animal Behaviour Science 58:213-220.

Ligout S, Bouissou M, Boivin X (2008) Comparison of the effects of two diferente handling methods on the subsequent behaviour of Anglo-Arabian foals toward humans and handling. Applied Animal Behaviour Science 113:175-188.

Lima RAS, Shirota R, Barros GSC (2006) Estudo do complexo do agronegócio cavalo. Coletânea Estudos Gleba 39 Piracicaba: CEPEA/ESALQ/USP.

Mackintosh NJ (1983) Conditioning and Associative Learning. Oxford: Claredon Press. 
McKinley J, Sambrook T (2000) Use of human-given cues by domestic dogs (Canis familiaris) and horses (Equus caballus). Animal Cognition 3:13-22.

Miller RM (1995) How the dominance hierarchy is determined: the body language of the horse. Journal of Equine Veterinary Science 15:514-515

De Passillé AM, Rushen J, Ladewig J, Petherick C (1996) Dairy calves' discrimination of people based on previous handling. Journal Animal Science 74:969-974.

Oliveira D (2003) Potenciais efeitos da estimulação tátil no comportamento e desenvolvimento de cordeiros e leitões. Tese, Universidade Estadual Paulista.

Oliveira D, Paranhos-da-Costa MJR, Zupan M, Rehn T, Keeling LJ (2015) Early human handling in non-weaned piglets: Effects on behaviour and body weight. Applied Animal Behaviour Science 164:56-63.

Seaman SC, Davidson HPB, Waran NK (2002) How reliable is temperament assessment in the domestic horse (Equus caballus)? Applied Animal Behaviour Science 78:175-191.

Simpson B (2002) Neonatal foal handling. Applied Animal Behaviour Science 78:303-317.

Søndergaard E, Halekoh U (2003) Young horses' reactions to humans in relation to handling and social environment. Applied Animal Behaviour Science 84:265-280.

Stone MS (2010) Human facial discrimination in horses: Can they tell us apart? Animal Cognition 13:51-61.

Tanida H, Miura A, Tanaka T, Yoshimoto T (1995) Behavioral response to humans in individually handled weanling pigs. Applied Animal Behaviour Science 42:249-259.

Tanida H, Koba Y (2001) How do miniature pigs discriminate between people? Applied Animal Behaviour Science 73:45-58.

Waiblinger S, Boivin X, Pedersen V, Tosi MV, Janczak AM, Visser EK, Jones RB (2006) Assessing the human-animal relationship in farm species: a critical review. Applied Animal Behaviour Science 101:185-242. 